\title{
Phase-sensitive near field Investigation of Bloch surface wave propagation in curved waveguides
}

\section{Wu}

\author{
E. Barakat \\ elsie.barakat@epfl.ch
}

L. Yu

L. Sun

J. Wang

Q. Tan

\section{H. P. Herzig}

Optics \& Photonics Technology Laboratory, Ecole Polytechnique Fédérale de Lausanne, EPFL-STI-OPT, CH-2000 Neuchâtel, Switzerland

Department of Precision Instruments, State Key Laboratory of Precision Measurement Technology and Instruments, Tsinghua University, Beijing 100084, China

Optics \& Photonics Technology Laboratory, Ecole Polytechnique Fédérale de Lausanne, EPFL-STI-OPT, CH-2000 Neuchâtel, Switzerland

Optics \& Photonics Technology Laboratory, Ecole Polytechnique Fédérale de Lausanne, EPFL-STI-OPT, CH-2000 Neuchâtel, Switzerland

Department of Precision Instruments, State Key Laboratory of Precision Measurement Technology and Instruments, Tsinghua University, Beijing 100084, China

Department of Precision Instruments, State Key Laboratory of Precision Measurement Technology and Instruments, Tsinghua University, Beijing 100084, China

Department of Precision Instruments, State Key Laboratory of Precision Measurement Technology and Instruments, Tsinghua University, Beijing 100084, China

Optics \& Photonics Technology Laboratory, Ecole Polytechnique Fédérale de Lausanne, EPFL-STI-OPT, CH-2000 Neuchâtel, Switzerland

Bloch surface waves (BSWs) are electromagnetic surface waves excited in the band gap of a one dimensional dielectric photonic crystal. They are confined at the interface of two media. Due to the use of dielectric material, the losses are very low, which allows the propagation of BSWs over long distances. Another advantage is the possibility of operating within a broad range of wavelengths. In this paper, we study and demonstrate the propagation of light in ultra-thin curved polymer waveguides having different radii fabricated on a BSWs sustaining multilayer. A phase-sensitive multi-parameter near-field optical measurement system (MH-SNOM), which combines heterodyne interferometry and SNOM, is used for the experimental characterization. Propagating properties, bending loss, mode conversion and admixture are investigated. We experimentally show that when light goes through the curved part of the waveguide, energy can be converted into different modes. The superposition and interference of different modes lead to a periodically alternating bright and dark beat phenomenon along the propagation direction. Experimental optical phase and amplitude distributions in the curved waveguide show a very good agreement with simulation results.

[DOI: http://dx.doi.org/10.2971/jeos.2014.14049]

Keywords: Bloch surface wave, curved waveguide, SNOM, mode conversion, phase detection

\section{INTRODUCTION}

Bloch surface waves (BSWs) are electromagnetic waves can be sustained on the surface of periodic layered media [1]. Compared with surface plasmon polaritions, BSWs shows lower absorption and allows larger spectral tenability [2]. In the past decade, BSW multilayer platform has been applied in two dimensional (2D) integrated optics and sensing application [3]- [5]. In a recent work, L. Yu et al. proposed a BSWssustaining multilayer as a platform for the integration of planar photonic components. By patterning optical elements on the top of the platform, BSWs can be deflected, diffracted, and focused [6]. Among these optical elements, bending curvature is one of the most basic elements in integrated circuits. Although bending loss caused by the curvature of waveguides has been studied for a long time [7]-[10], the subject is still of high interest for surface plasmon polariton [11]-[13] and Bloch surface waves [6]. Therefore, in this paper, we experimentally and theoretically investigate the propagation of
BSWs in bended waveguides. Investigations on its energy loss and mode distribution and conversion have a guiding significance on integrated photonics design. In order to have a better understanding of light properties of these optical elements, a Phase-sensitive Multi-Heterodyne Scanning near-field optical microscope (MH-SNOM) combines optical heterodyne interferometry and SNOM is induced. As already known, SNOM have the optical resolution beyond the diffraction limit and can realize sub-wavelength imaging of optical intensity distribution which has been widely used in integrated photonics [14]-[16]. Using conventional commercial SNOMs, phase information is lost during the measurements, only topography and optical intensity distribution can be obtained. However, compared with the optical intensity, phase is a more fundamental physical parameter.

It can reveal the nature of light and interactions between light and matter, especially in nanophotonics [17]-[19]. MH- 


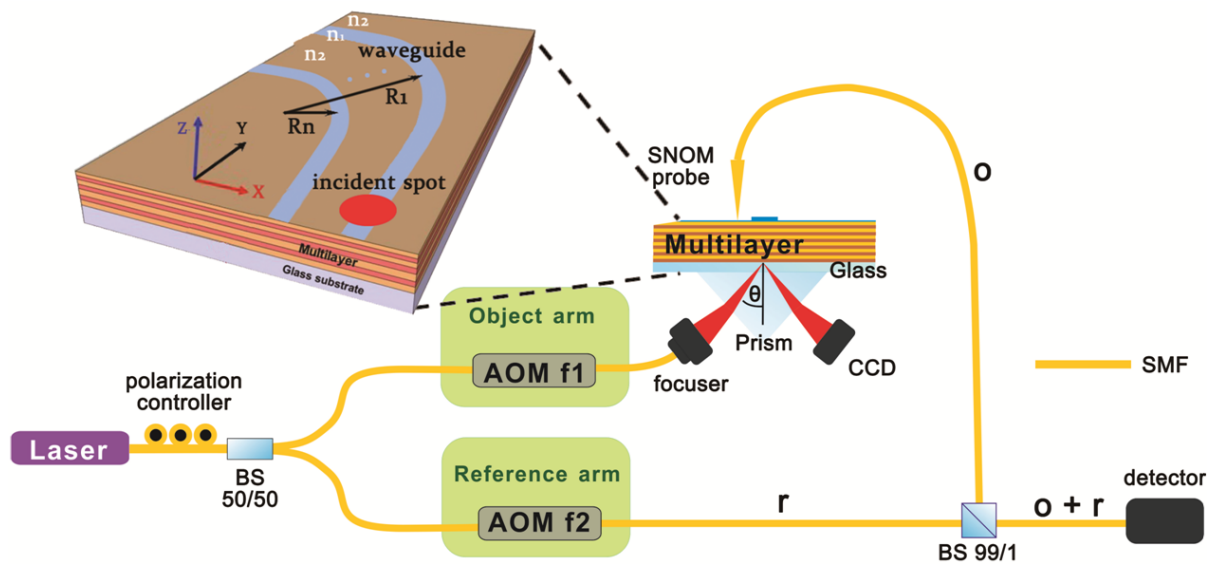

FIG. 1 Schematic of the multi-heterodyne near field optical microscope (MH-SNOM) measurement. BSWs are excited in the Kretchmann configuration and propagate in the waveguides. The inset shows the multilayer, polymer waveguides which are fabricated on the top of the multilayer.

SNOM can simultaneously measure amplitude, phase and polarization in the near field. Therefore, it is used in this paper in order to study two dimensional polymer bending waveguides excited with surface electromagnetic waves.

\section{EXPERIMENTAL SETUP}

Bloch surface waves are excited in the band gap of a one dimensional photonic crystal made of a dielectric multilayer that alternates high and low refractive indices. The multilayer is fabricated by chemical vapor deposition (PECVD), where six periods of $\mathrm{Si} 3 \mathrm{~N} 4$ and $\mathrm{SiO} 2$ are alternately deposited on a glass substrate. The silicon nitride and silicon oxide thicknesses are $263 \mathrm{~nm}$ and $492 \mathrm{~nm}$ respectively. An additional 80 $\mathrm{nm}$ thick layer of silicon nitride is deposited on the multilayer. The total thickness of the multilayer is about $4 \mu \mathrm{m}$. The thickness of the top layer defines the location of the dispersion line in the band gap, consequently, the properties of the propagation mode on the platform. On the top of it, $100 \mathrm{~nm}$ thick dielectric waveguides made of positive photoresist (AZ1518) are fabricated. These waveguides are $3 \mu \mathrm{m}$-wide and their radius varies between $18 \mu \mathrm{m}$ and $80 \mu \mathrm{m}$. The Bloch surface waves are excited under the condition of total internal reflection as shown in Figure 1. A focused laser beam, at lambda $=1562 \mathrm{~nm}$, illuminates the lower surface of the multilayer. The coupled spot of the BSWs on the surface of the multilayer has a diameter of $30 \mu \mathrm{m}$. After passing through the multilayer, the incident beam should match the propagation constant $\beta=k \sin \theta$ in order to allow the excitation and propagation of BSWs in the $x-y$ plane. $k=n_{g} \cdot 2 \pi / \lambda$ is the wave vector of the incident beam, $n_{g}$ is the refractive index of glass, and $\theta$ is the incident angle. BSWs are excited and propagate along the waveguide $[4,20]$. Thus, for a constant wavelength, we can deduce the effective index of the BSW for both uncoated and PRcoated platform. At $\lambda=1542 \mathrm{~nm}$, the effective index of the waveguide mode in bare multilayer is $n_{M L}=1.1862$ while the effective index in the $100 \mathrm{~nm}$ thick additional layer of photoresist $(\mathrm{PR})$ is $n_{P R}=1.2415$. By adding a PR layer on the top of the platform, we introduce a refractive index contrast of the BSWs between the PR layer and the bare multilayer. In the case of $100 \mathrm{~nm}$-thick layer of PR, the refractive index contrast is $\Delta n=n_{P R}-n_{M L}=0.055$.
The schematic of the phase sensitive MH-SNOM system is shown in Figure 1. Two arms form an interferometer, one is the reference, and the other is the object arm which carries information from the sample. Two acousto-optic modulators $(\mathrm{AOM})$ are used to create a frequency difference between the object and the reference channels. This leads to a $20 \mathrm{kHz}$ beat frequency signal that is detected by detectors. The optical signals are collected by an Al-coated SNOM probe (aluminized fiber probe with a diameter about $200 \mathrm{~nm}$ ). The interference signal from the two arms is detected and sent to a lock-in amplifier. With the demodulation of the lock-in amplifier, both the optical amplitude and phase from the sample measurement can be extracted [3]. Since phase is much more sensitive to the environmental change than intensity. Some methods must be taken to suppress the phase drift and disturbance. To decrease the phase drift, optical length of object arm and reference arm should be equal. Larger optical path difference lead to larger phase drift when disturbed by external environment. Minimize the optical path helps increase system stability. To decrease the noise disturbance, all the optical fibers and electric cables are covered with anechoic sponge and capsulated in a plexiglass box.

\section{RESULTS AND DISCUSSION}

We first start by studying the straight part of the waveguide. Figure 2(a) shows the intensity distribution measured by the SNOM. BSWs are coupled into the polymer waveguide and propagate along the y-axis. The measured intensity distribution shows that the transmission efficiency in the straight waveguide is about $94 \%$ per $100 \mu \mathrm{m}$. This loss is mainly due to the leakage back into the multilayer (design) and due to scattering (fabrication technology).

The waveguide theory, described in reference [21], shows that the number of modes $(m)$ that can exist in the waveguide is determined by the wavelength, the width of the waveguide, the refractive index of waveguide material and the surrounding materials as follows:

$$
\tan \left(\pi \frac{a}{\lambda} \sin \theta-m \frac{\pi}{2}\right)=\left(\frac{\sin ^{2} \bar{\theta}_{c}}{\sin ^{2} \theta}-1\right)^{1 / 2}
$$


(a)

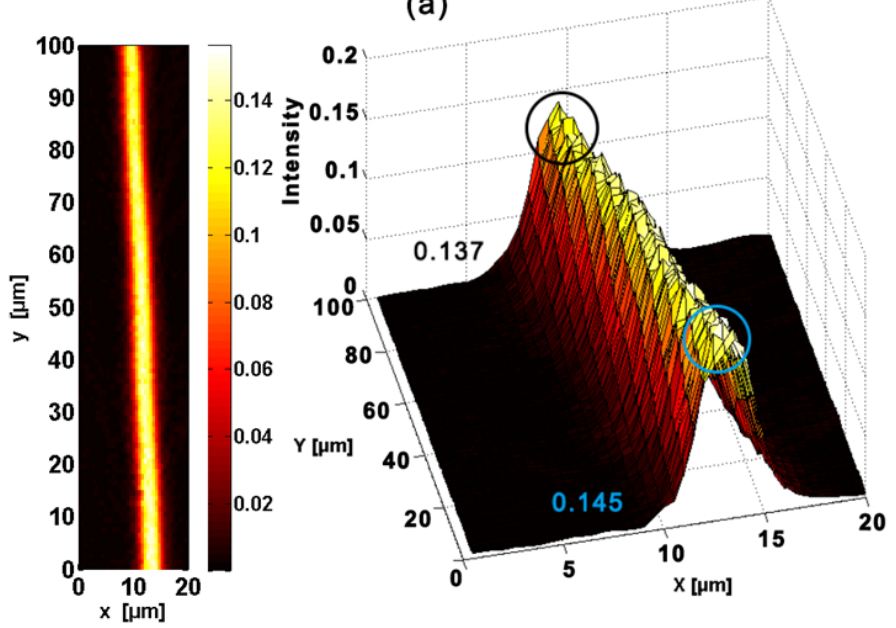

(b)

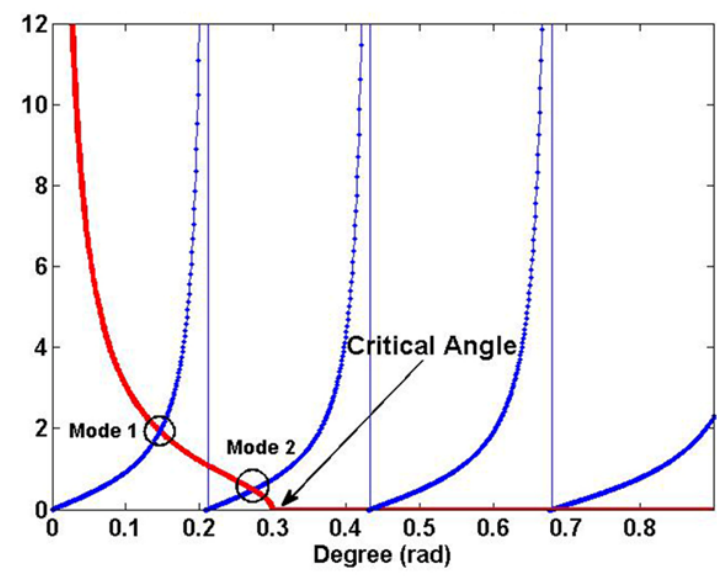

FIG. 2 (a) Experimental intensity distribution of a $3 \mu \mathrm{m}$-wide straight waveguide measured by a MH-SNOM. (b) Graphical solutions of waveguide modes. The red and blue intersections circled in (b) stand for all the possible modes in the waveguide.
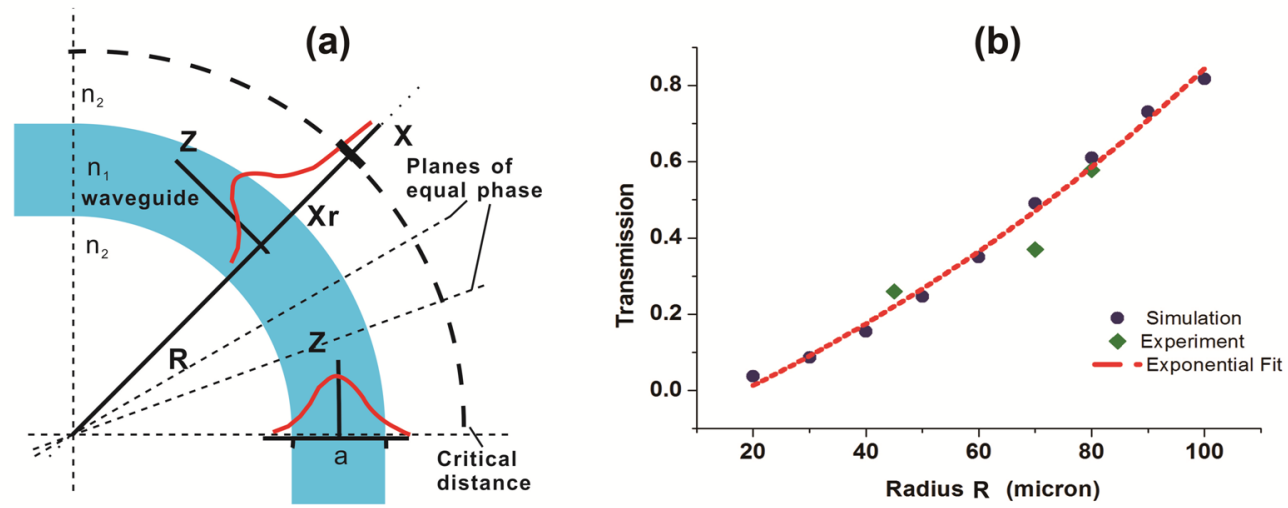

FIC. 3 (a) Field distribution in a curved waveguide. (b) Transmission as a function of the curvature radius $R$. The red line is an exponential fit of simulated points which shows that the transmission is exponentially growing with the radius $R$. Green points stand for the measured radii.

Where $a$ is the waveguide's width, and $\bar{\theta}_{c}$ is the critical angle. This is a transcendental equation with one variable $\sin \theta$. Its solutions yield the angles $\theta_{m}$ of the modes. Figure 2(b) shows the graphical solution of Eq. (1). The intersections in Figure 2(b) stand for all the possible modes that can propagates in the waveguide at a certain wavelength. The red line corresponds to the right part while the blue line corresponds to the left part. As one can see, a $3 \mu \mathrm{m}$ - wide polymer waveguide is multimode around a wavelength of $1.56 \mu \mathrm{m}$ where two modes can be excited. This means that both radiation and mode conversion losses have to be considered in the total bending loss $[22,23]$. Therefore for a better understanding of the experimental result, we will briefly describe in the next section bending and mode conversion losses in optical waveguides.

\subsection{Bending loss}

When electromagnetic waves propagate in bended waveguides, a part of the energy will be lost by radiation. This radiation loss may be only negligible if the curvature radius of the waveguide is sufficiently large. Several mathematical proof have been reported in the literature [23, 24]. A slab bending waveguide is presented in Figure 3. The refractive index of the waveguide $n_{1}$ is larger than that of the surrounding region $n_{2}$, resulting to a transverse field distribution $F(x)$ that decays exponentially. $R$ is the radius of waveguide curvature. When the light propagates along the waveguide, a part of the energy propagates in the outer region. Close to the waveguide axis, the phase front moves at a velocity equal to that in the straight waveguide. However, in the medium outside the waveguide, the velocity is slower than the velocity of plane waves. It is clear that the phase velocity in the surrounding medium away from the center of curvature $(x>R)$ is higher than the phase velocity in the waveguide, in order to preserve the phase front [25]. At a certain distance away from the waveguide $x_{r}$, the phase velocity equals the plane wave velocity in the surrounding medium. The electromagnetic wave cannot move faster than the characteristic velocity in the particular medium. The energy in the guided mode at $x>x_{r}$ is assumed to be lost by radiation. This explains why bent dielectric waveguides must lose power by radiation [26].

Figure $3(b)$ shows the transmission as a function of the curvature radius $R$. It is clear that the transmission increases by increasing the radius $R$ due to the decrease of the radiation losses. The transmission is defined as the ration of the intensity at the end of the bent waveguide over the intensity at 


\section{Experimental}

Results

(a)

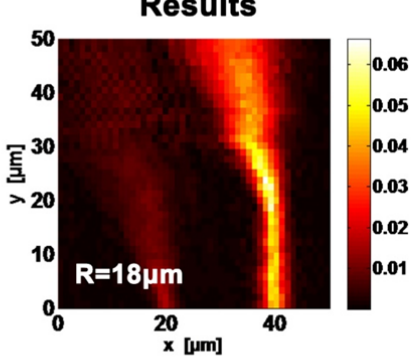

(b)

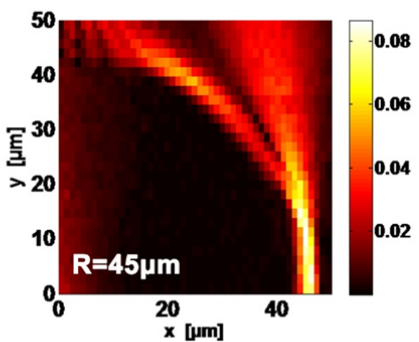

(c)

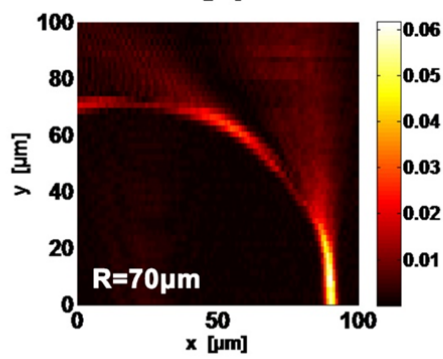

(d)

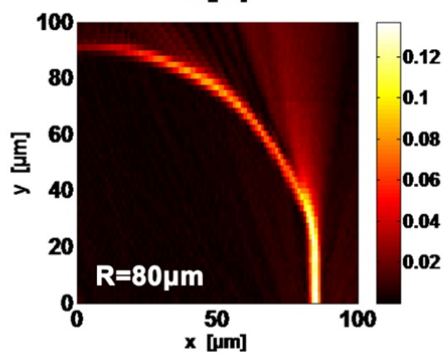

Simulation

Results
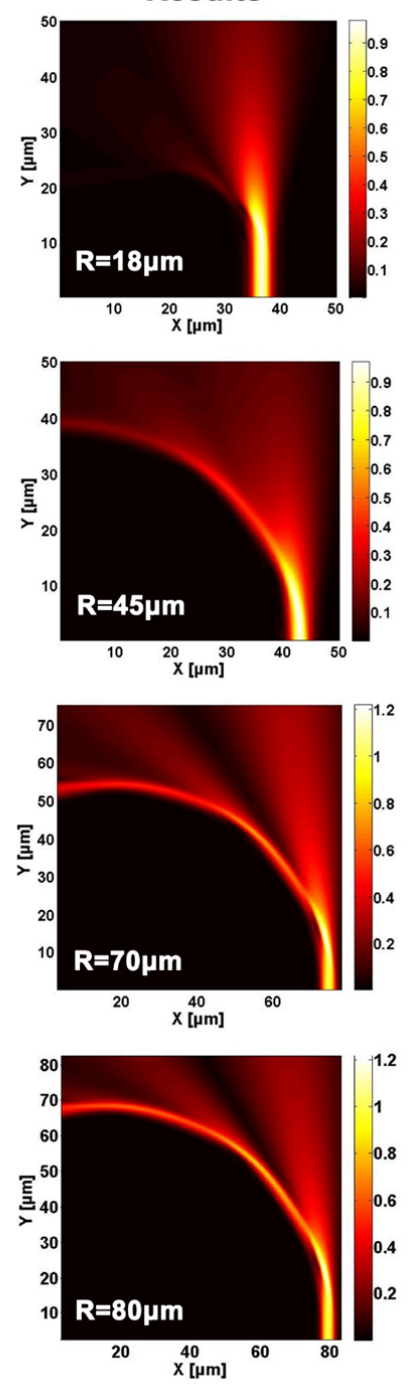

FIC. 4 Experimental (left) and theoretical (right) intensity distribution $\left(\left|E_{x}\right|^{2}+\left|E_{y}\right|^{2}\right)$ of bending waveguides having different radii values. (a) $R=18 \mu \mathrm{m}$, (b) $R=45 \mu \mathrm{m}$, (c) $\mathrm{R}=70 \mu \mathrm{m},(\mathrm{d}) \mathrm{R}=80 \mu \mathrm{m}$.

the beginning of the bent region. Experimental results (Green Square) in Figure 3(b) show good agreement with the simulation (blue dots) using the finite difference time domain method (FDTD). For a curvature radius of $80 \mu \mathrm{m}$, losses remain small and the corresponding transmission is of $60 \%$ for a 100nm-thick layer of PR. The thickness of the PR plays a key role in the efficiency of bended curves since it is directly related to the effective index contrast, $\Delta n$. By increasing the $\mathrm{PR}$ thickness, the refractive index contrast increases leading to a decrease of the critical radius which is important for the miniaturization of future designs.

\subsection{Conversion of bending modes}

Although the bending loss is mainly caused by radiation loss for single mode waveguides, the mode conversion also has a great effect on the bending loss in multimode waveguides. Defects or abruptly bent of the waveguide can lead to mode conversion loss [23]. The dielectric tensor as a function of space can be written as: $\vec{\epsilon}(x, y, z)=\epsilon_{a}(x, y, z)+\Delta \epsilon(x, y, z)$ where $\epsilon_{a}(x, y, z)$ is the unperturbed part of the dielectric tensor, and $\Delta \epsilon(x, y, z)$, represents the dielectric perturbation due to waveguide imperfections, bending, surface corrugations, or the like. When there is a dielectric perturbation $\Delta \epsilon(x, y, z)$, the propagation modes are coupled to each other. In other words, if a pure mode is excited at the beginning of the waveguide, some of its power may be transferred to other modes [27]. In case of an unperturbed propagation mode, $E_{1}(x, y, z) e^{i\left(\omega t-\beta_{1} z\right)}$, the presence of the dielectric $\Delta \epsilon(x, y, z)$ gives rise to an additional polarization

$$
\Delta \vec{P}=\Delta \epsilon(x, y, z) \vec{E}_{1}(x, y, z) e^{i\left(\omega t-\beta_{1} z\right)}
$$

If the polarization wave, acting as a radiating source, can feed energy into (or out of) some other mode $E_{2}(x, y, z) e^{i\left(\omega t-\beta_{2} z\right)}$, then we say $E_{1}$ and $E_{2}$ modes couple to each other and cause energy exchange. When light goes from the straight waveguide to the curved region, the radius is suddenly changed from infinity to $R$. The fundamental mode in the straight waveguide will excite other modes [22]. The mode conversion coefficient between the fundamental and the first order modes which is proportional to $1 / R$, indicates that for smaller radius, greater energy might be converted.

\subsection{Near field experiments}

Figure 4 shows a comparison of near field measurement and theoretical field distributions of bending waveguides excited by BSWs. The studied radii are 18, 45, 70, and $80 \mu \mathrm{m}$. For a curvature of $18 \mu \mathrm{m}$, both theory and experiment show that light is tangentially leaked from the bending waveguide. The phase fronts at the outside of the bent waveguide start to bend. The phase velocity would have to exceed the plane wave velocity in the medium outside the waveguide to keep a flat phase front. Since this condition is not possible, the field bends and starts radiating into space. For a curvature of $45 \mu \mathrm{m}$, leaked light decreases and part of the injected BSWs propagates inside the bending waveguide. The transmission of a $45 \mu \mathrm{m}$ curved waveguide is about $26 \%$. For 70 and $80 \mu \mathrm{m}$ curvature, energy propagate along the bended waveguide where alternating bright and dark fringes, forming a beat, appear in the field distribution. The maximum absolute value of the electric field shifts towards the outer rim (rim with the larger radius) of the bend. If the refractive index difference between waveguide and surrounding media decrease, the relative field levels in the exterior region increase.

When a fundamental mode light propagates from straight waveguide to the curved region, the radius is suddenly changed from infinity to $R$, which can be considered as a dielectric perturbation $\Delta \epsilon(x, y, z)$. From Eq. (2) previously discussed, part of energy will be coupled to other modes. In a dielectric waveguide, the propagation constant $\beta$ for each mode is a function of $\omega$. It is because the interference and superposition of different modes lead to a beat phenomenon. In the exterior region, the mode interference pattern radiates and diverges rapidly. The direction of the light leakage is tangential to the curved waveguide, starting from the maximum intensity of the outer rim. These are caused by the superposition interference of the two modes [28]. The smaller the radius is, the greater the loss of energy. Actually, when the radius is larger than $80 \mu \mathrm{m}$, the loss caused by radiation 

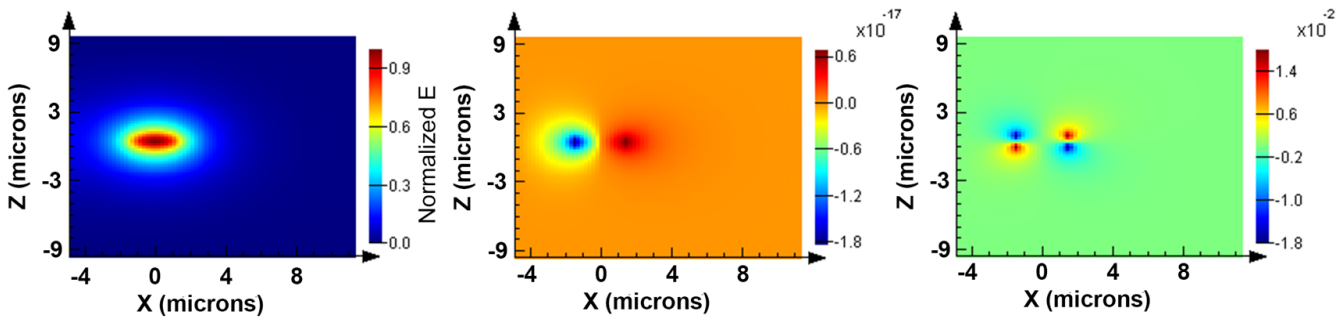

FIG. 5 Amplitude distribution of the three Cartesian components of the electric field inside the waveguide at $1.562 \mu \mathrm{m}$ in the $\mathrm{xz}$-plane at $\mathrm{y}=0$.

(a)

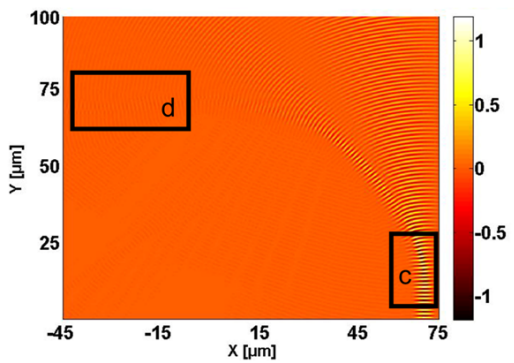

(c)

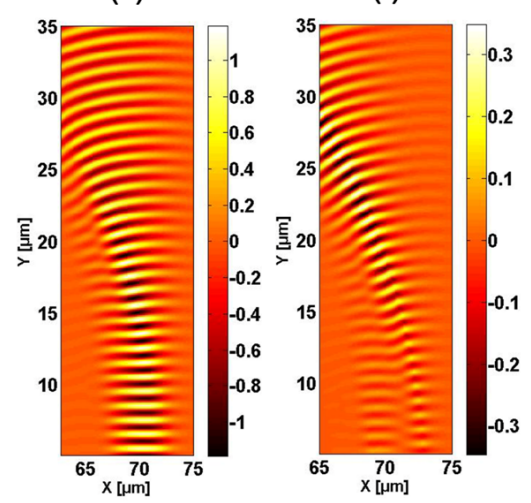

(b)

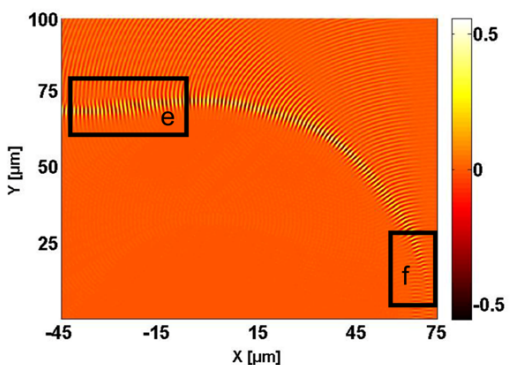

(d)

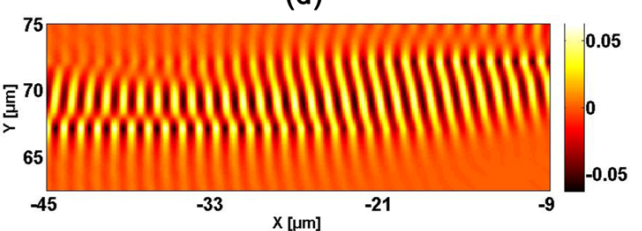

(e)

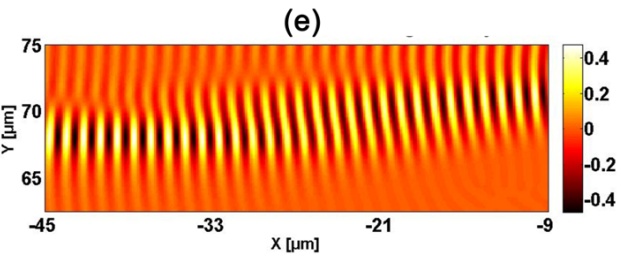

FIG. 6 Mode conversion and coupling in the curved waveguide simulated by FDTD. (a) and (b) show the real part of the $E_{x}$, $E_{y}$ components of a $70 \mu m$ bending waveguide, respectively. Figure $6(\mathrm{c}),(\mathrm{d}),(\mathrm{e})$ and $(\mathrm{f})$ are enlarged parts mentioned in the black boxes in Figure $6(\mathrm{a})$ and (b). Figure $6(\mathrm{c})$ and $6(\mathrm{f})$ shows the $E_{x}$ and $E_{y}$ components are fundamental mode and first order mode, respectively. While Figure 6(d) and 6(e) reveals new mode are excited when passing through the curvature, different modes co-exist and interference with each other.

is small. In this case, the loss is mainly due to the mode conversion. It is also important to note that for the current design of the multilayer and the used polymer, a refractive index contrast between the multilayer and the waveguide is small. This value can be increased by using other materials for the waveguide fabrication. In this latter case, the curvature showing negligible loss might be decreased.

Figure 5 shows the field distribution of the three components of the electric field of the fundamental mode in a $3 \mu \mathrm{m}$-wide waveguide. The total energy is normalized and the relative electric field intensity of $\mathrm{x}, \mathrm{y}, \mathrm{z}$ components are distributed. The $E_{x}$ component plays a dominant role of the mode, while the $E_{y}$ and $E_{z}$ components are weak compared to the $E_{x}$ component which can be regarded as zero. In order to observe the mode conversion clearly, a simulation of the curved waveguide is completed by the FDTD method. As we know the aperture probe mainly be sensitive to the electric field within the sample plane. So, the incident polarization, simulation and measurement are all based on this plane. Figure 6(a) and 6(b) show the real part of the $E_{x}, E_{y}$ components of a $70 \mu \mathrm{m}$ bending waveguide, respectively. At straight part, the $E_{x}$ components remain dominant while the $E_{y}$ component is dominant in the curved region. Figure 6(c), (d), (e) and (f) are enlarged parts mentioned in the black boxes in Figure 6(a) and 6(b).

Figure 6(c) and 6(f) shows the $E_{x}$ and $E_{y}$ components are fundamental mode and first order mode, respectively. Initially, $E_{y}$ is quite weak compared with the $E_{x}$ component before propagating into the curved region. It is worth to note that we can observe a $\pi$ phase shift between the inner and outer modes in the waveguide. After entering the bend region, the optical path difference of the two modes changes and phase become equal. The constructive interference induced by phase matching enhances the energy of the $E_{y}$ component. The strengthened energy comes from the decreased part of the $E_{x}$ component. When the light leaves the curved area, another new 
(a)

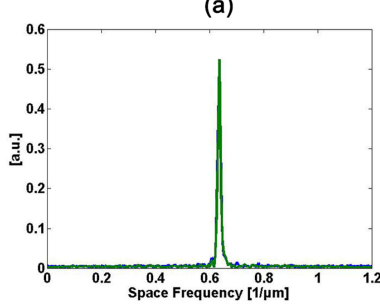

(b)

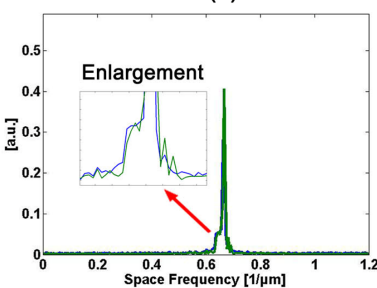

(c)

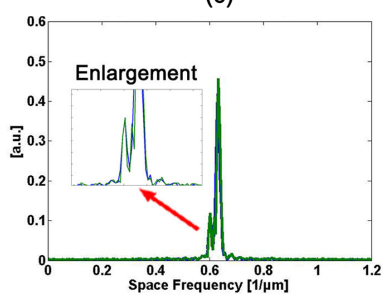

FIG. 7 Two-dimension FFT on the complex near-field measured with the SNOM at different positions. (a) is the FFT result in the straight waveguide, (b) is the FFT result in the bend region while $(c)$ is the FFT result at the end of the bend region. The green and blue line stand for two measurements.

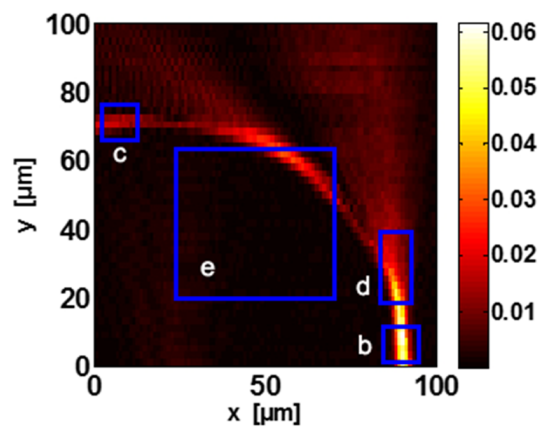

(a)

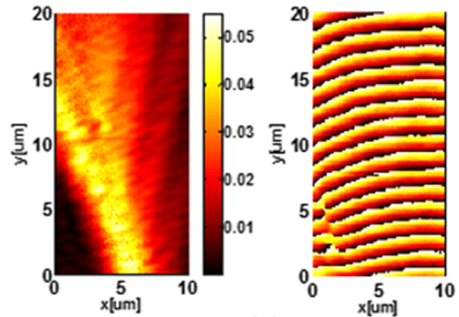

(d)
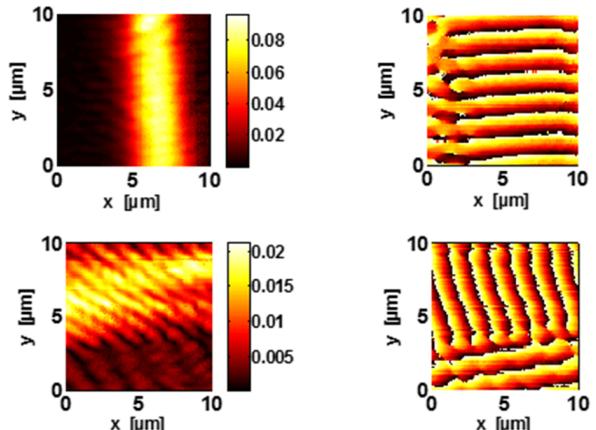

(b)
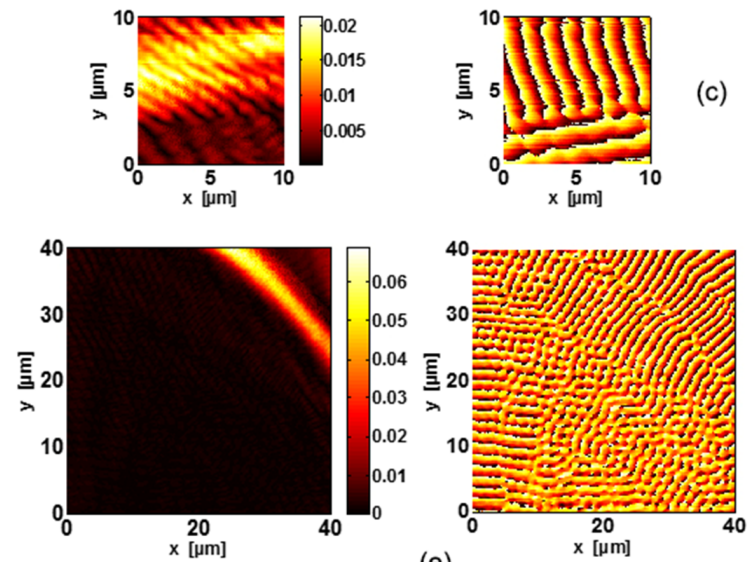

(e)

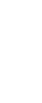

FIG. 8 optical intensity and phase distributions in the waveguide having a curvature of $70 \mu \mathrm{m}$ are measured by a MH-SNOM. The total intensity image of a bended waveguide is shown in Figure 8 (a). The 4 squares in Figure $8(a)$ indicate the areas where the corresponding enlargements are provided in Figure 8 (b) $\sim(e)$ with the intensity and phase measurements, respectively.

mode is generated. The original mode and the new excited mode co-exist and aliasing appears, as shown in 6(d) and 6(e).

\subsection{Phase distribution}

Although the mode propagation and conversion can reveal and explain some mechanism of propagation in the bending waveguide, the phase distribution will lead to a deeper understanding of light propagation in the waveguide. Mapping the phase distribution is a powerful method to investigate propagation features, like mode conversion. MH-SNOM gives the phase and amplitude distributions, leading a measurement of the optical real field $A e^{i \phi}$, in which $A$ and $\phi$ stand for optical amplitude and phase, respectively. In order to study the mode propagation in the bend region, a fast Fourier transforms is performed. Different peaks in spectrum stand for different spatial frequencies and different modes. The single peak in Figure 7(a) is obtained in the straight waveguide before entering the curvature indicating a fundamental mode. $\beta_{0}=2 \pi / \lambda_{0}=2 \pi k_{0}, k$ is the spatial frequency. When the radius is suddenly changed from infinity to $R$, the fundamental mode in the straight waveguide will excite other modes which exhibit different space frequency. Figure 7(b) reveals a tendency to two peaks. It indicates that there is a mode conversion at the beginning of the bend region. The double peaks in Figure 7(c) stand for fundamental and first order mode aliasing. Figure 7(c) is obtained at the end of the curvature. It is clear that after going through the bend waveguide, a new mode is excited and propagates in the waveguide.

The measured optical intensity and phase distributions in a waveguide having a curvature of $70 \mu \mathrm{m}$ are shown in Figure 8 . The total intensity image of a bended waveguide is shown in Figure 8(a). The 4 squares in Figure 8(a) indicate the areas where the corresponding enlargements are provided with the intensity and phase measurements, respectively. The phase fronts in Figure 8(b) show that the fundamental mode is excited in the straight part of the waveguide. Figure 8(c) shows the intensity and the phase distribution of light at the exit straight waveguide after crossing the bending area. For distances smaller than the radius, the phase front remains perpendicular to the waveguide. This is due to the propagation of uncoupled light in the waveguide. Figure 8(d) shows the characteristics of light at the beginning of the bending area where a high leakage is seen from the waveguide. At a position larger than the critical radius, the phase velocity there would have to 
exceed the characteristic velocity in the medium outside the waveguide to keep a flat phase front. Since this condition is not possible, the field bends and starts radiating into space. Figure 8(e) shows that in the region smaller than radius, the energy remains weak, while the phase distribution shows that light is clearly propagating inside the waveguide. It performs a scaly like phase interference pattern in the central part of the curvature, which is contributed by different positions in the waveguide. Since their direction of propagation is different, the superposition of their phase fronts results in the scaly pattern.

\section{CONCLUSION}

This paper demonstrates and investigates the propagation of Bloch surface waves, excited in total internal reflection configuration, in bended polymer-based waveguides. We mainly discuss the bending loss, mode conversion and optical field distribution of curved waveguides. For this particular configuration, we started with calculating a 2D curved waveguide model and experimental measurements. The simulations are then discussed in order to illustrate the physical behavior that influences the bending loss. The light outside the waveguide has an equal phase front with the light in the waveguide. However, its velocity can't be greater than the characteristic velocity in a particular medium. Thus, at a position larger than the critical radius, light can't be guided and loss increases due to high radiation losses. Another part of the energy losses is due to mode conversion and admixture when light goes through the curved part of the waveguide. The superposition and interference of different modes lead to a periodically alternating bright and dark beat phenomenon along the propagation direction in experimental results. In order to verify that the beat phenomenon is not due to sample defects, a FDTD simulation is induced as a contrast. The simulation results explain the energy leakage in tangential direction of the curvature and beat phenomenon. With the phase and amplitude distributions obtained by MH-SNOM, we analyze the optical field by the Fast Fourier Transform in order to investigate the optical real field to investigate the new excited modes. Phase measurement is a powerful tool for us to analyze field distribution of planar optical elements.

\section{ACKN OWLEDGMENTS}

This research is supported by the Swiss National Science Foundation (SNSF FN200020-135455) and National Natural Science Foundation of China. (Grant No. 61177089 and No. 61227014)

\section{References}

[1] P. Yeh, A. Yariv, and A. Y. Cho, "Optical surface waves in periodic layered media," Appl. Phys. Lett. 32, 104-105 (1978).

[2] C. Vandenbem, "Electromagnetic surface waves of multilayer stacks: coupling between guided modes and Bloch modes," Opt. Lett. 33, 2260-2262 (2008).
[3] T. Sfez, E. Descrovi, L. Yu, D. Brunazzo, M. Quaglio, L. Dominici, W. Nakagawa, et al., "Bloch surface waves in ultrathin waveguides: near-field investigation of mode polarization and propagation," J. Opt. Soc. Am. B 27, 1617-1625 (2010).

[4] E. Descrovi, T. Sfez, M. Quaglio, D. Brunazzo, L. Dominici, F. Michelotti, H. P. Herzig, et al., "Guided Bloch Surface Waves on Ultrathin Polymeric Ridges," Nano Lett. 10, 2087-2091 (2010).

[5] A. Angelini, E. Barakat, P. Munzert, L. Boarino, N. De Leo, E. Enrico, F. Giorgis, H. P. Herzig, et al., "Focusing and Extraction of Light mediated by Bloch Surface Waves," Sci. Rep. 4, (2014).

[6] L. Yu, E. Barakat, T. Sfez, L. Hvozdara, J. Di Francesco, and H. Peter Herzig, "Manipulating Bloch surface waves in 2D: a platform concept-based flat lens," Light Sci. Appl. 3, e124 (2014).

[7] M. E. Marhic, "Mode-coupling analysis of bending losses in IR metallic waveguides," Appl. Optics 20, 3436-3441 (1981).

[8] E. A. J. Marcatili, and S.E. Miller, "Improved Relations Describing Directional Control in Electromagnetic Wave Guidance," Bell Syst. Tech. J. 48, 2161-2188 (1969).

[9] H. F. Taylor, "Losses at corner bends in dielectric waveguides," Appl. Optics 16, 711-716, (1977).

[10] M. Heiblum and J. Harris, "Analysis of curved optical waveguides by conformal transformation," IEEE J. Quantum Elect. 11, 75-83 (1975).

[11] Z. Zhu, C. E. Garcia-Ortiz, Z. Han, I. P. Radko, and S. I. Bozhevolnyi, "Compact and broadband directional coupling and demultiplexing in dielectric-loaded surface plasmon polariton waveguides based on the multimode interference effect," Appl. Phys. Lett. 103, 061108-5 (2013).

[12] L. Zhang, J. Yang, X. Fu, and M. Zhang, "Graphene disk as an ultra compact ring resonator based on edge propagating plasmons," Appl. Phys. Lett. 103, 163114-5 (2013).

[13] K. Hassan, A. Bouhelier, T. Bernardin, G. Colas-des-Francs, J. C. Weeber, A. Dereux, and R. Espiau de Lamaestre, "Momentumspace spectroscopy for advanced analysis of dielectric-loaded surface plasmon polariton coupled and bent waveguides," Phys. Rev. B 87, 195428 (2013).

[14] D. Courjon, and C. Bainier, "Near field microscopy and near field optics," Rep. Prog. Phys. 57, 989 (1994).

[15] S. Kawata, Y. Inouye, and P. Verma, "Plasmonics for near-field nano-imaging and superlensing," Nat. Photonics 3, 388-394 (2009).

[16] L. Novotny, "The history of near-field optics," in Progress in Optics, E. Wolf, ed., 137-184 (Elsevier, Amsterdam, 2007).

[17] T. SFEZ, Investigation of Surface Electromagnetic Waves with Multi-Heterodyne Scanning Near-Field Optical Microscopy, (PhD Dissertation, École Polytechnique Fédérale de Lausanne, 2010).

[18] P. Tortora, Optical Properties of Nano-Structured Materials Studied by Means of Interferometric Techniques (PhD Dissertation, Universiity of Neuchatel, 2005).

[19] P. S. Carney, B. Deutsch, A. A. Govyadinov, and R. Hillenbrand, "Phase in Nanooptics," ACS Nano 6, 8-12 (2012).

[20] T. Sfez, E. Descrovi, L. Yu, M. Quaglio, L. Dominici, W. Nakagawa, F. Michelotti, et al., "Two-dimensional optics on silicon nitride multilayer: Refraction of Bloch surface waves," Appl. Phys. Lett. 96, 151101-151103 (2010).

[21] B. E. A. Saleh, and M. C. Teich, Fundamentals of Photonics (Wiley, Hoboken, 2013). 
[22] S. Kawakami, "Mode conversion losses of randomly bent, singly and doubly clad waveguides for single mode transmission," Appl. Optics 15, 2778-2784 (1976).

[23] E. G. Neumann, "Curved dielectric optical waveguides with reduced transition losses," Microwaves, Optics and Antennas, IEE Proceedings H 129, 278-280 (1982).

[24] R. G. Hunsperger, Integrated Optics: Theory and Technology (Springer, Berlin, 2009).

[25] D. Marcuse, "Bending Losses of the Asymmetric Slab Waveguide," Bell Syst. Tech. J. 50, 2551-2563 (1971).
[26] R. W. Neumann, "Sharp bends with low losses in dielectric optical waveguides," Appl. Optics 22, 1016-1022 (1983).

[27] A. Yariv, and P. Yeh, Photonics: Optical Electronics in Modern Communications (0xford University Press, 0xford, 2007).

[28] K. R. Hiremath, M. Hammer, R. Stoffer, L. Prkna, and J. Čtyroký, "Analytic approach to dielectric optical bent slab waveguides," Opt. Quant. Electron. 37, 37-61 (2005). 